\title{
Development of an experimental rig for soil and crop residues management
}

\begin{abstract}
The development of an experimental rig was performed to modified blades geometry and lifting angles for the suitability of pulverization of oil palm fronds to reduce torque, noise and vibration and fuel consumption during field operations. The experimental rig and the blades were attached to the soil bin and the transducers were connected to the data acquisition module. The blades were attached to the blade carrier and the appropriate operated speed was selected via the control box. Three level of mulching depth $(10,15$ and $30 \mathrm{~cm})$ were compared based on the level of pulverisation on the four blades. Sieve analysis was carried out to determine the level of pulverisation. The statistical analysis is based on ANOVA test of significance based on completely randomized design (CRD). The model showed significance $(\mathrm{P}>0.05)$ on the depth of operations but insignificant between the blades and pulverisation level and attributable with the design model having $\mathrm{R} 2=0.44$. It is expected that more models are needed to test the fitness of the designed blades.
\end{abstract}

Keyword: Experimental rig; Mulcher blades; Crop residues; Depth and pulverisation 